\title{
Carbohydrate derived fulvic acid: an in vitro investigation of a novel membrane active antiseptic agent against Candida albicans biofilms
}

\section{Leighann Sherry ${ }^{1}$, Anto Jose $^{1}$, Colin Murray ${ }^{1}$, Craig Williams ${ }^{2}$, Brian Jones ${ }^{3}$, Owain Millington ${ }^{4}$, Jeremy Bagg ${ }^{1}$ and Gordon Ramage ${ }^{1 *}$}

\author{
'Infection and Immunity Research Group, Glasgow Dental School, School of Medicine, College of Medical, Veterinary and Life Sciences, University of Glasgow, \\ Glasgow, UK \\ ${ }^{2}$ Microbiology Department, Royal Hospital for Sick Children (Yorkhill Division), Glasgow, UK \\ ${ }^{3}$ Microbiology Department, Glasgow Royal Infirmary, Glasgow, UK \\ ${ }^{4}$ Strathclyde Institute of Pharmacy and Biomedical Sciences, University of Strathclyde, Glasgow, UK
}

\author{
Edited by: \\ Fernando César Bizerra, Federal \\ University of São Paulo, Brazil \\ Reviewed by: \\ Dmitri Debabov, NovaBay \\ Pharmaceuticals, USA \\ Sueli Fumie Yamada-Ogatta, \\ Universidade Estadual de Londrina, \\ Brazil

\section{*Correspondence:} \\ Gordon Ramage, Infection and \\ Immunity Research Group, Glasgow \\ Dental School, School of Medicine, \\ College of Medical, Veterinary and \\ Life Sciences, University of Glasgow, \\ 378 Sauchiehall Street, Glasgow G2 \\ 3JZ, UK. \\ e-mail: gordon.ramage@ \\ glasgow.ac.uk
}

\begin{abstract}
Carbohydrate derived fulvic acid (CHD-FA) is a heat stable low molecular weight, water soluble, cationic, colloidal material with proposed therapeutic properties. The aim of this study was to evaluate the antifungal activity of CHD-FA against Candida albicans, and to characterize its mode of action. A panel of $C$. albicans isolates $(n=50)$ derived from a range of clinical specimens were grown planktonically and as biofilms, and the minimum inhibitory concentrations determined. Scanning electron microscopy was performed to examine ultrastructural changes and different cell membrane assays were used to determine its mode of action. In addition, the role of $C$. albicans biofilm resistance mechanisms were investigated to determine their effects on CHD-FA activity. CHD-FA was active against planktonic and sessile $C$. albicans at concentrations 0.125 and $0.25 \%$ respectively, and was shown to be fungicidal, acting through disruption of the cell membrane activity. Resistance mechanisms, including matrix, efflux, and stress, had a limited role upon CHD-FA activity. Overall, based on the promising in vitro spectrum of activity and minimal biofilm resistance of the natural and cheap antiseptic CHD-FA, further studies are required to determine its applicability for clinical use.
\end{abstract}

Keywords: Candida albicans, fulvic acid, biofilm, antiseptic

\section{INTRODUCTION}

Oral diseases such as oral candidosis, caries, and periodontitis are characterized by microbial biofilms (Coco et al., 2008), which are difficult to control with standard chemotherapeutic approaches and host immune defenses (Ramage et al., 2010). These biofilm consortia have a community structure, dominant metabolic processes and inter-organism interactions. Antimicrobial resistance is a key characteristic of biofilms, which is associated with exopolymeric matrix, increased cell density, persister cells, and up-regulation of efflux pumps (Ramage et al., 2009). Recent studies by our group demonstrated that Candida albicans biofilms were refractory to prescription antifungal agents, mouthwashes, and denture cleansers (Jose et al., 2010; Ramage et al., 2011a). In addition, Candida biofilms in the oral cavity are associated with inflammation and symptoms such as pain, burning sensation, and altered taste (Samaranayake et al., 2009). Overall, these factors complicate clinical management, therefore, alternative agents that elicit antifungal activity are of clinical interest.

Fulvic acid is a novel antimicrobial molecule that is reported to have antibacterial and antifungal properties (van Rensburg et al., 2000). Moreover, it has been recently reported to be non-toxic in a rat wound model, in addition to having anti-inflammatory properties (Sabi et al., 2011). A recent randomized, double blind, controlled trial indicated that fulvic acid was well-tolerated in a study of eczema (Gandy et al., 2011). This colloidal organic acid is a major constituent of humic acids and has been recognized for its biological significance for many years, yet there is minimal scientific understanding on which to support the audacious claims of its properties. Fulvic acid can be isolated from the environment or produced from the oxidation of coal or lignite. Such preparations contain high levels of heavy metals and potentially toxic elements, making their use in humans unsuitable. Recent innovation has seen the development of carbohydrate derived fulvic acid (CHDFA), a pure form of fulvic acid produced by a patented process to GMP standards (PA107470/GB), rendering it free of heavy metals and environmental pollutants normally found in fulvic acid from environmental sources.

The purpose of this study was to investigate the antifungal effects of CHD-FA, to determine whether it was active against biofilms and to define its mode of action. We report for the first time a highly effective novel antiseptic agent with exquisite biofilm activity that acts by disrupting cell membranes.

\section{MATERIALS AND METHODS CULTURE CONDITIONS AND STANDARDIZATION}

Candida albicans type strains ATCC 90028, ATCC 10231, 3153A, SC5314, CAF 2, and a range of clinical strains $(n=45)$ were used for sensitivity testing. All working stocks of $C$. albicans were 
maintained at $4^{\circ} \mathrm{C}$ on Sabouraud (SAB; Oxoid, Cambridge, UK) agar. Isolates were propagated in yeast peptone dextrose (YPD) medium (Oxoid), washed by centrifugation, and resuspended in RPMI-1640, as described previously (Ramage et al., 2001).

\section{ANTIFUNGAL SUSCEPTIBILITY TESTING OF PLANKTONIC CELLS AND BIOFILMS}

The following antifungal agents were used in the course of this study: CHD-FA [Fulhold, Cape Town, South Africa (CAS: 479-663)], voriconazole (VRZ, Pfizer Pharmaceuticals, Sandwich, UK), caspofungin (CSP, Merck Sharp \& Dohme, Hertfordshire, UK), amphotericin B (AMB, Sigma, Poole, UK).

Antifungal testing to determine minimum inhibitory concentrations (MICs) of planktonic cells was performed using the CLSI M-27A broth microdilution method (CLSI, 2008). Biofilms were formed and sessile susceptibility testing was performed as previously described in commercially available presterilized, polystyrene, flat-bottomed, 96-well microtiter plates (Corning Incorporated, NY, USA; Ramage et al., 2001). Sessile minimum inhibitory concentrations (SMICs) were determined at $80 \%$ inhibition using an XTT [2,3-bis(2-methoxy-4-nitro-5sulfo-phenyl)-2H-tetrazolium-5-caboxanilide] metabolic reduction assay (Pierce et al., 2008). Testing of all planktonic and sessile isolates was performed in triplicate.

For time-kill studies four isolates were selected based on their superior and equivalent biofilm properties. These were grown and treated with CHD-FA, VRZ, CSP, and AMB at 1,2, and $4 \times \mathrm{SMIC}_{50}$ to investigate whether these antifungals work in a dose dependent manner. At 1, 2, 4, 6, 8, and $24 \mathrm{~h}$ the biofilms were carefully washed and the metabolic activity of the biofilms assessed (Pierce et al., 2008). Testing of four isolates for each drug and each time-point was performed with six replicates and on two separate occasions.

\section{BIOFILM DISRUPTION AND ULTRASTRUCTURAL CHANGES}

Candida albicans biofilms $(n=40)$ were prepared and treated with 2 and $4 \times$ SMIC $_{50}$ for $24 \mathrm{~h}$ as these concentrations of CHD-FA were highly effective in the time-kill study above. Following treatment the wells were washed carefully with PBS to quantify the biomass of each biofilm, as previously reported using a crystal violet assay (Jose et al., 2010).

For scanning electron microscopy C. albicans 3153A sessile cells were grown directly onto Thermanox ${ }^{\mathrm{TM}}$ coverslips (Nunc, Roskilde, Denmark) prior to antifungal treatment, whereas planktonic $C$. albicans cells were first pretreated with CHD-FA prior to immobilization on the coverslip. Treatment was performed with all four antifungal agents for $24 \mathrm{~h}$ at $1 \times$ SMIC $_{90}$ for both planktonic and sessile cells. These were then fixed in $2 \%$ para-formaldehyde, $2 \%$ glutaraldehyde and $0.15 \mathrm{M}$ sodium cacodylate, and $0.15 \% \mathrm{w} / \mathrm{v}$ Alcian Blue, $\mathrm{pH} 7.4$, and prepared for SEM as previously described (Erlandsen et al., 2004). The specimens were sputter-coated with gold and viewed under a JEOL JSM-6400 scanning electron microscope. Images were assembled using Photoshop software (Adobe, San Jose, CA, USA).

\section{MECHANISM OF ACTION}

We investigated whether CHD-FA interacted with the membrane using a propidium iodide (PI) uptake and ATP release assay.
First we standardized C. albicans (SC5314) to $5 \times 10^{7}$ cells $/ \mathrm{ml}$ in RPMI-1640 and treated with CHD-FA at 4\% for 10, 20, 30, 40, 50 , and $60 \mathrm{~min}$. Following treatment, cells were washed twice with PBS, stained with $20 \mu \mathrm{M}$ of PI and incubated at $37^{\circ} \mathrm{C}$ for $15 \mathrm{~min}$ to allow the dye to bind to DNA. Fluorescence was then measured at excitation and emission wavelengths $535 / 617 \mathrm{~nm}$, respectively. Alternatively, cells in RPMI were plated onto an Ibidi $\mu$ Slide VI (Thistle Scientific, Glasgow, UK) and cultured for $60 \mathrm{~min}$ at $37^{\circ} \mathrm{C}$ before imaging on an inverted Nikon Eclipse TE300 using a $\times 20$ objective lens.

ATP release was also quantified from collected supernatants. These were buffered to $\mathrm{pH} 7.0$ and equal volumes of supernatant were added to ATP assay mix (Sigma-Aldrich, UK), and the assay performed as per the manufacturer's instructions. Luminescence was then measured in a white 96-well plate (Corning Incorporated, NY, USA) using a microtiter plate reader (FLUOStar Omega, BMG Labtech). Membrane assays were carried out in triplicate and repeated at least twice.

We next investigated the contribution of chitin, using a specific inhibitor of the chitin synthesis pathway \pm CHD-FA, to delineate its mechanism of action. For planktonic cells CLSI methodologies were employed, with CHD-FA \pm nikkomycin $\mathrm{Z}(0.4 \mu \mathrm{g} / \mathrm{ml})$, which was incubated for $24 \mathrm{~h}$ at $37^{\circ} \mathrm{C}$. Sessile cells were grown in the presence of nikkomycin $\mathrm{Z}$ for $24 \mathrm{~h}$ at $37^{\circ} \mathrm{C}$ then treated with CHD-FA for another $24 \mathrm{~h}$. Following incubation of both planktonic cells and biofilms the metabolic activity was quantified using the XTT reduction assay (Pierce et al., 2008). This assay was carried out on two individual occasions, using two type and two clinical strains tested in quadruplicate.

\section{RESISTANCE MECHANISMS}

The role of heat shock protein 90 (Hsp90) was investigated using a specific inhibitor of the Hsp90 (17-allylamino-17demethoxygeldanamycin) \pm CHD-FA. Planktonic cells and preformed biofilms were treated with $\mathrm{CHD}-\mathrm{FA} \pm$ geldanamycin $\left(12.5 \mu \mathrm{g} / \mathrm{ml}\right.$; Invivogen, San Diego, CA, USA) for $24 \mathrm{~h}$ at $37^{\circ} \mathrm{C}$. Following incubation the metabolic activity of the cells was quantified using the XTT reduction assay (Pierce et al., 2008). This assay was carried out on two individual occasions, using two type and two clinical strains tested in quadruplicate.

The role of extracellular matrix was investigated using strains previously described with altered expression of FKS1 (Nett et al., 2010a). Reference strain (DAY185), FKS1/fks1 $\Delta$ and TDH3-FKS1 were standardized to $1 \times 10^{6}$ cells $/ \mathrm{ml}$ in 96 -well flat-bottomed plates and biofilms grown for $8 \mathrm{~h}$ at $37^{\circ} \mathrm{C}$. Biofilms were carefully washed three times with PBS and treated with 0.05 and $0.1 \%$ CHDFA for $24 \mathrm{~h}$. After incubation, biofilms were carefully washed three times with PBS and metabolic activity was measured using the XTT assay (Pierce et al., 2008). This assay was carried out on two separate occasions, in triplicate.

Efflux pump activity of planktonic and biofilm cells was initially assessed using an MC-005,556 (Ala-Nap) fluorescent assay (Ramage et al., 2011b). C. albicans SC5314 was prepared as standardized planktonic cells $\left(\sim 5 \times 10^{7}\right.$ cells $\left./ \mathrm{ml}\right)$ in buffer solution $\left[\mathrm{K}_{2} \mathrm{HPO}_{4}\right.$ $(50 \mathrm{mM}), \mathrm{MgSO}_{4}(1 \mathrm{mM})$, and glucose $\left.(0.4 \%)\right]$ at $\mathrm{pH}$ 7.0. Planktonic cells were treated with 0.0313 and $0.0625 \%$ CHD-FA for 4 and $24 \mathrm{~h}$ before being washed with PBS, resuspended in assay 
buffer and added to black flat-bottomed microtiter plate (Costar 3603, Corning, NY, USA). Biofilms were grown directly within the plates and treated with CHD-FA for 4 and $24 \mathrm{~h}$. After incubation, biofilms were washed and the buffer solution added. The reaction was initiated by the addition of Ala-Nap at a final concentration of $100 \mu \mathrm{g} / \mathrm{ml}$. Fluorescence was quantified at $30 \mathrm{~s}$ intervals for $1 \mathrm{~h}$ at $37^{\circ} \mathrm{C}$ using a fluorescence plate reader (FLUOstar OPTIMA) at excitation and emission wavelengths of $355 / 460 \mathrm{~nm}$, respectively. Higher fluorescence values indicated low efflux activity and vice versa. To determine whether efflux pump activity was detrimental to the effects of CHD-FA, an efflux pump inhibitor [EPI; LPhe-L-Arg- $\beta$-naphthylamide (MC-207,110), Sigma-Aldrich] was prepared at a working concentration in distilled water (Ramage et al., 2011b). To determine the effects of MC-207,110 on the MIC of CHD-FA, a checkerboard titration assay was performed. CHD-FA was tested at a range of $0.002-1 \%$ in combination with MC-207,110 at a concentration of $64 \mu \mathrm{g} / \mathrm{ml}$. Viability was assessed using the XTT reduction assay as previously described (Pierce et al., 2008).

\section{STATISTICAL ANALYSIS}

Data distribution and statistical analysis was performed using GraphPad Prism (version 4; La Jolla, CA, USA). The nonparametric data was analyzed using the Mann-Whitney $U$ test to assess differences between two independent sample groups. Statistical significance was achieved if $p<0.05$.

\section{RESULTS}

\section{CHD-FA EXHIBITS POTENT ANTIFUNGAL ACTIVITY}

All planktonic C. albicans isolates tested were susceptible to all four antifungal drugs, with $\mathrm{MIC}_{90}$ for CHD-FA, VRZ, AMB, and CSP of $0.125 \%$ and $0.125 \mathrm{mg} / \mathrm{l}$, respectively (Table 1). $\mathrm{MIC}_{50}$ and $\mathrm{MIC}_{90}$ refer to the MICs of $\geq 50 / 90 \%$ of isolates inhibited by each antifungal, respectively. When the sessile activity of each compound was compared to planktonic cells in terms of $\mathrm{MIC}_{90}$ fold change, CHD-FA was the most effective $(2 \times)$, followed by caspofungin $(4 \times)$, amphotericin B $(64 \times)$ and voriconazole $(>2048 \times)$. Both planktonic and sessile cells showed comparable MIC's for all 50 strains tested.

The biofilm rate of kill of CHD-FA was assessed, where dose and time dependent killing was observed (Figure 1Ai). Greater killing was observed after 1,2 , and $4 \mathrm{~h}$ at $4 \times \mathrm{SMIC}_{50}$ in comparison to 1 and $2 \times \mathrm{SMIC}_{50}$ where a decrease in viability of 65 and $89 \%$ was observed. After $24 \mathrm{~h}$ treatment no differences were observed between each concentration, with an approximate final $92 \%$ kill. When each antifungal was compared at $4 \times \mathrm{SMIC}_{50}$ then $\mathrm{CHD}$ FA displayed the quickest killing compared in ascending order to $\mathrm{AMB}, \mathrm{VRZ}$ and CSP, which after $2 \mathrm{~h}$ was $89,61,18$, and $-2 \%$, respectively (Figure 1Aii). However, after $24 \mathrm{~h}$ the most effective compound was CSP (97\%) followed by CHD-FA (91\%), AMB (88\%), and VRZ (43\%).

The ability to disrupt C. albicans biofilms was then evaluated, where it was shown that both $0.25 \%$ and $0.5 \%$ CHD-FA were unable to significantly reduce the biofilm biomass compared to a PBS control ( $p=0.2318$ and 0.1069 , respectively; Figure 1B).

\section{CHD-FA IS NON-SPECIFICALLY ACTIVE AGAINST CELL MEMBRANES}

Deterioration of the general cell structure of both planktonic and sessile cells was observed, of which the latter appear to be crenated (Figure 2A). Based on these observations it was hypothesized that the cell membrane may have been destabilized, so membrane permeability assays were performed. ATP was released in a time dependent manner, reaching a maximum after $40 \mathrm{~min}$ exposure to CHD-FA (Figure 2B). These observations were confirmed through quantification of PI, where uptake was shown to be rapid over the first $20 \mathrm{~min}$, after which this plateaued. Using time-lapse microscopy, uptake of PI was shown to occur rapidly following addition of CHD-FA, with detectable membrane permeability within $10 \mathrm{~min}$ of exposure to CHD-FA, with all cells becoming PI-positive and cell growth attenuated (Figures 2Ci,ii).

To further elucidate the mechanisms of action we investigated the impact of chitin using its inhibitor nikkomycin (NKM). We hypothesized that by weakening the chitin layer this would enable CHD-FA to have greater activity, if indeed the cell membrane was the target. C. albicans planktonic cells treated with $0.0625 \%$ CHD-FA in the presence of NKM were found to be significantly more sensitive to CHD-FA $(p=0.0022)$ than NKM free cells (Figure 2D), which is demonstrated by a $21 \%$ reduction in cell viability. Treatment of $C$. albicans biofilm showed a $\sim 34 \%$ reduction in cell viability, however, this was not statistically significant $(p=0.1057)$. In both planktonic and sessile states, NKM showed no effect on cell viability (data not shown).

Table 1 | Susceptibility profile of Candida albicans to four antifungal agents.

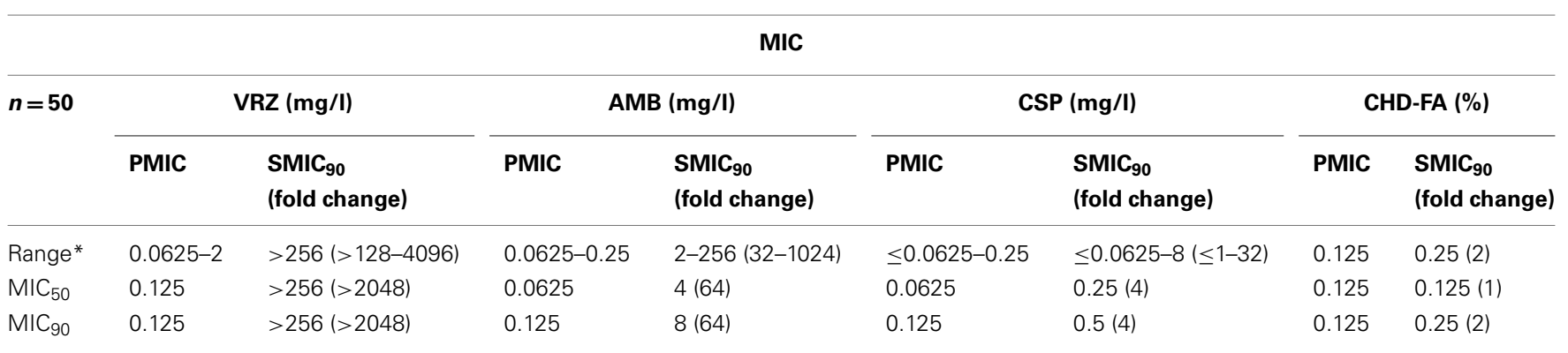

PMIC, planktonic minimum inhibitory concentration; SMIC, sessile minimum inhibitory concentration; $S M I C_{90}$, $90 \%$ reduction in viability assessed by $X T T$; VRZ, voriconazole (VFend® - Pfizer); AMB, amphotericin B (Sigma-Aldrich); CSP, caspofungin (Cancidas $®$ - Merck), CHD-FA, carbohydrate derived fulvic acid (Fulhold). ${ }^{*}$ MIC range of all isolates. 

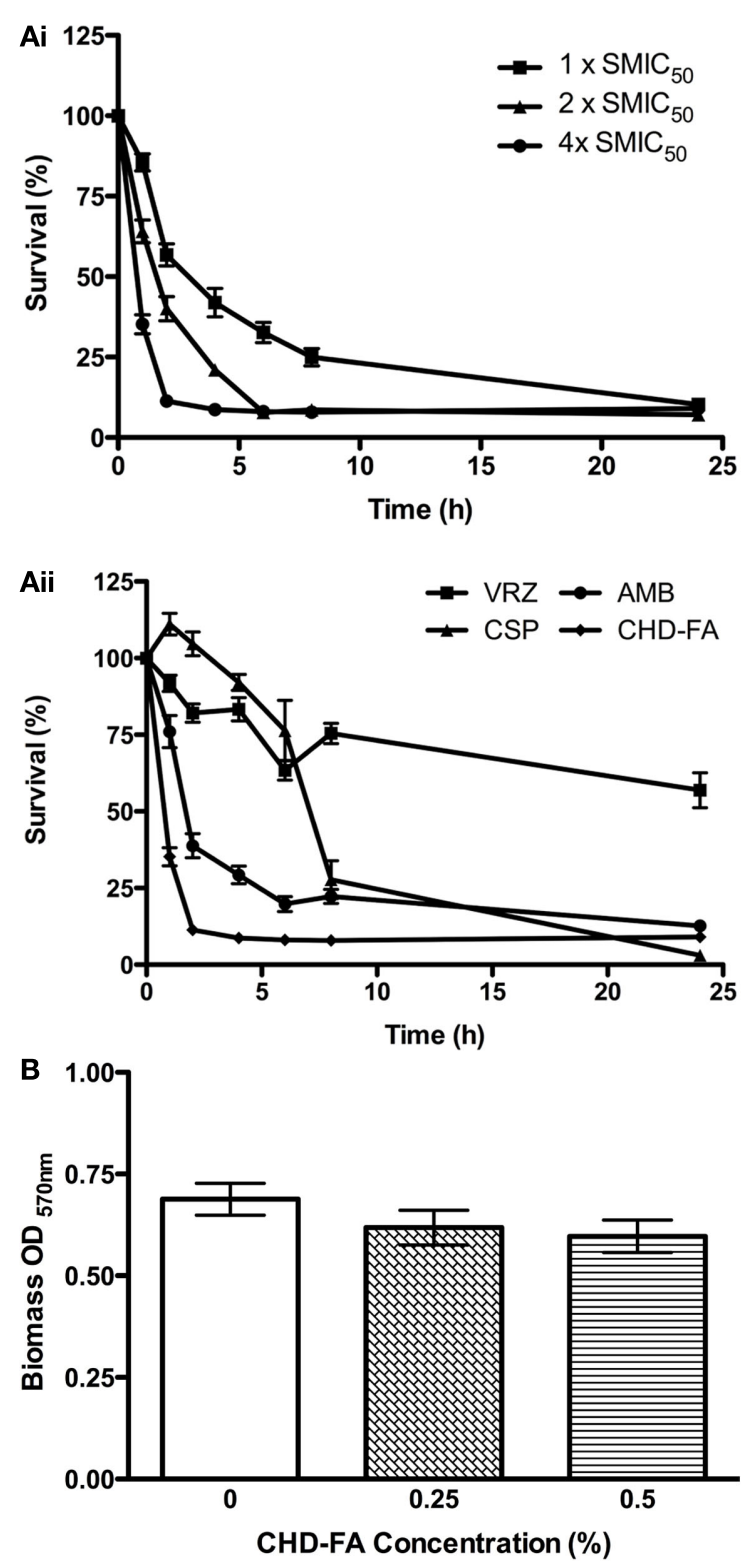

FIGURE 1 | Candida albicans biofilm killing by CHD-FA is time and concentration dependent, despite no biofilm disruption. (A) Biofilm time-kill kinetics of CHD-FA at 1,2 , and $4 \times \mathrm{SMIC}_{50}$. (i) Biofilm time-kill kinetics of VRZ, CSP, AMB and CHD-FA at $4 \times S M I C_{50}$. (ii) Standardized C. albicans $\left(1 \times 10^{6}\right.$ cells $\left./ \mathrm{ml}\right)$ were grown in flat-bottomed 96 -well plates for $24 \mathrm{~h}$, washed in PBS and treated with antifungal agents at defined concentrations for $1,2,4,6,8$, and $24 \mathrm{~h}$. Metabolic activity of treated biofilms was then quantified using the XTT assay. Four isolates were used for each assay, and this was performed on two independent occasions in triplicate. (B) Standardized C. albicans $\left(1 \times 10^{6} \mathrm{cells} / \mathrm{ml}\right)$ were grown in flat bottomed 96-well plates for $24 \mathrm{~h}$, washed in PBS and treated with 0.25 and $0.5 \% \mathrm{CHD}-\mathrm{FA}$ for $24 \mathrm{~h}$. A negative control was also included. The biomass of the biofilm was then quantified by staining each biofilm with $0.05 \% \mathrm{w} / \mathrm{v}$ crystal violet solution. The biofilms were washed, allowed to air dry, and $100 \%$ ethanol applied to destain each biofilm. The biomass was quantified spectrophotometrically by reading at $570 \mathrm{~nm}$ in a microtiter plate reader (FLUOStar Omega, BMG Labtech). Forty isolates were tested during this assay, which was performed in triplicate.

\section{THE ROLE OF CANDIDA ALBICANS BIOFILM RESISTANCE}

\section{MECHANISMS}

The roles of key resistance mechanisms utilized by C. albicans were next investigated to determine whether they were likely to impact the effectiveness of CHD-FA. C. albicans susceptibility to $0.0625 \%$ CHD-FA (sub-MIC) in the presence of the Hsp90 inhibitor geldanamycin (GDM) was significantly increased in planktonic $(p=0.0009)$ and sessile $(p=0.0133)$ cells (Figure 3A), reducing the cell viability by 49 and $7 \%$, respectively. No significant differences were observed for both planktonic and sessile cells treated at MIC levels $(0.125 \%)$ in the presence of GDM. In both cell states, GDM showed no effect on cell viability (data not shown).

The role of FKS1 expression in C. albicans biofilm resistance was assessed. Figure 3B shows that FKS1/fks $1 \Delta$ biofilms were significantly more susceptible to CHD-FA at sub-MIC levels $(0.05 \%)$ compared to the treated reference strain $(p=0.0126)$, with cell viability being reduced by $40 \%$. In comparison, the over-expressing strain, TDH3-FKS1, was less sensitive to $0.05 \%$ CHD-FA, with cell survival $10 \%$ greater than the reference strain $(p=0.3095)$.

Carbohydrate derived fulvic acid treated planktonic cells exhibited a time and dose dependent up-regulation of efflux pumps compared to untreated cells (Figure 3Ci). As the CHD-FA concentration increased ( 0 to 0.0313 and $0.0625 \%$ ) there was significant up-regulation of efflux pumps, indicated by low fluorescence, after treatment for $4 \mathrm{~h}(p=<0.0001)$, but not after $24 \mathrm{~h}(p=0.2581$ and 0.0625 , respectively). For sessile cells a general increase in efflux pump activity was observed compared to planktonic cells. However, no significant increase in efflux pump activity was reported when the biofilms were exposed for $4 \mathrm{~h}$ to 0.0313 and $0.0625 \%$ CHD-FA ( $p=0.3401$ and 0.0503 respectively). Conversely, $24 \mathrm{~h}$ treated biofilms showed a minor down-regulation of efflux pumps compared to the untreated control at these concentrations ( $p=0.0003$ and 0.0019 , respectively; Figure 3Cii). In addition to efflux pump activity being measured, the effects of an EPI on sensitivity were explored (Figure 3Ciii). There was no significant reduction in cell survival when $0.0625 \%$ CHD-FA and EPI were used in combination $(p=0.0569)$, despite a $10 \%$ reduction in viability. EPI showed no effect on cell viability (data not shown).

\section{DISCUSSION}

Many oral diseases are associated with microbial biofilms, which are generally refractory to prescription antibiotics and antifungal agents. Recently we reported that commercial mouthwashes were superior to these and a more favorable chemotherapeutic option for treating oral candidal biofilm infections (Ramage et al., 2011a). However, even with chlorhexidine containing products residual viable biofilm cells are retained, so novel compounds are still urgently required. Here we report for the first time a potential novel antimicrobial agent, CHD-FA, which has potent antifungal activity against $C$. albicans biofilms and other oral biofilms. Furthermore, we propose that this compound is effective through disruption of the cellular membrane and is unaffected by biofilm resistance mechanisms. 
A i

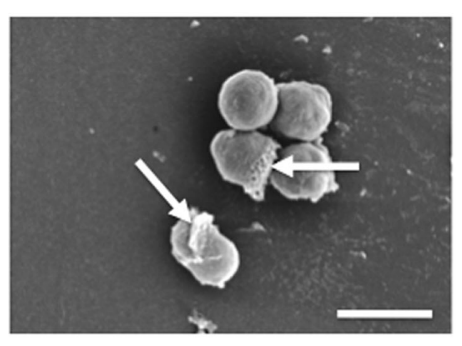

ii

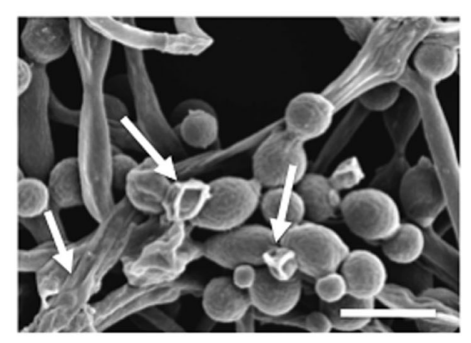

B

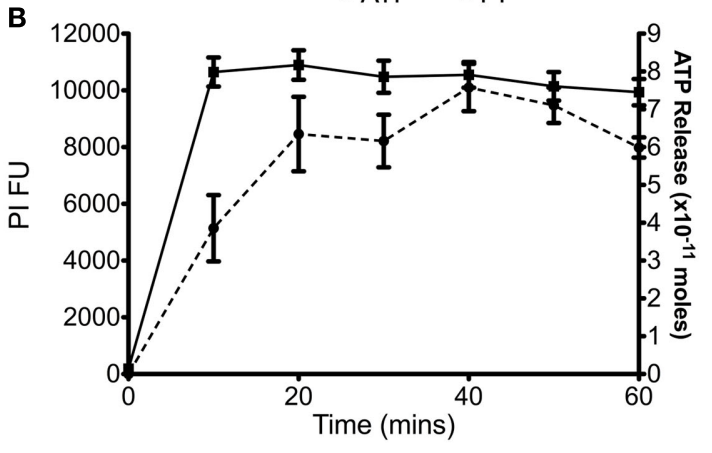

Cii

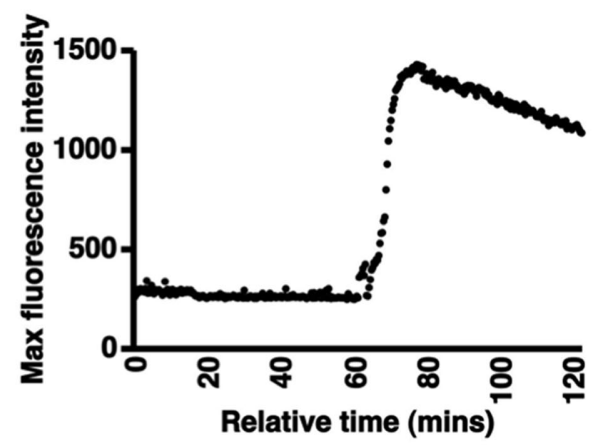

Ci
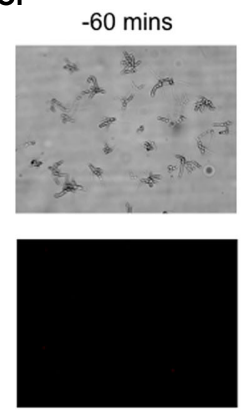

0 mins
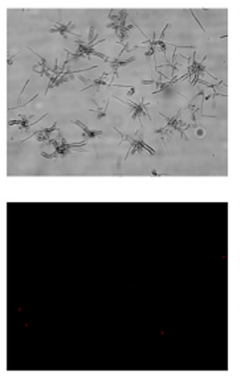

$10 \mathrm{~min}$
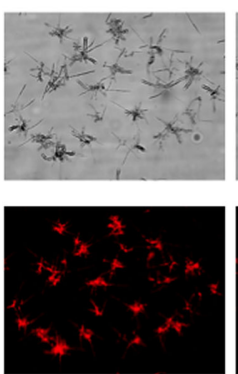

60 mins
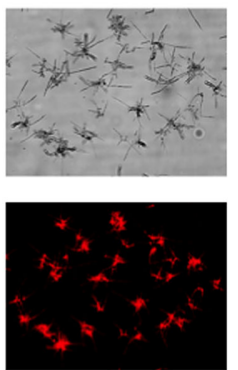

D

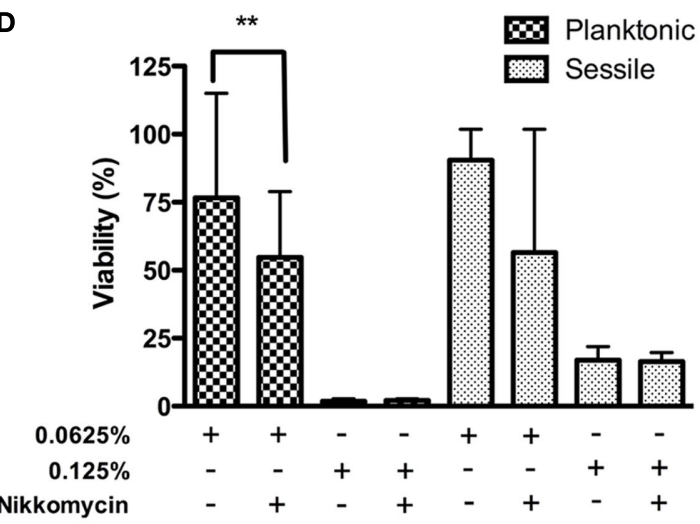

FIGURE 2 | Carbohydrate derived fulvic acid permeabilizes Candida albicans cell membranes. (A) Planktonic cells (i) and sessile (ii) cells were treated at their respective $\mathrm{MIC}_{90}$ for $24 \mathrm{~h}$ either in solution or on

Thermanox ${ }^{\mathrm{TM}}$ coverslips, respectively. These were then processed and viewed on a JEOL JSM-6400 scanning electron microscope and images assembled using Photoshop software. Note the apparent disruption of the cell wall denoted by arrows on both the planktonic and sessile cells. Scale bars represent $5 \mu \mathrm{m}$. (B) C. albicans (SC5314) planktonic cells

$\left(5 \times 10^{7} \mathrm{cells} / \mathrm{ml}\right)$ were treated with CHD-FA (4\%) for 10, 20, 30, 40, 50, and 60 min. A negative control (no CHD-FA) was also included. For PI experiments the cells were washed by centrifugation, resuspended in $\mathrm{PI}$ $\left(20 \mu \mathrm{M}\right.$ in PBS), and incubated for $15 \mathrm{~min}$ at $37^{\circ} \mathrm{C}$. These were then transferred to a black 96-well plate for quantification in a fluorescent plate reader $\left(E x_{485} / E m_{620}\right)$. For ATP release the cells were removed by filter sterilization $(0.22 \mu \mathrm{m})$ and supernatants adjusted to a pH of $7.8 \mathrm{in} 10 \mathrm{mM}$
$\mathrm{NaOH}$. The assay, including standards, was performed as per manufacturer's instructions in a white well plate for quantification in a luminescent plate reader. Each assay was performed on at least two independent occasions in triplicate. (C) C. albicans cells were allowed to grow on an Ibidi $\mu$ Slide $V I$ for $60 \mathrm{~min}$ before $20 \mu \mathrm{M} \mathrm{PI}$ and $2 \%$ CHD-FA was added. PI uptake was measured over 60 min using time-lapse microscopy, with images taken every $20 \mathrm{~s}$. Note the sudden uptake of PI into $C$. albicans cells after exposure to CHD-FA for $10 \mathrm{~min}$ (i), which was validated through quantifiable data that showed a rapid increase in fluorescence when CHD-FA was added (ii). (D) C. albicans was grown as planktonic and biofilm cells \pm nikkomycin $Z$ $(0.4 \mu \mathrm{g} / \mathrm{ml})$ for $24 \mathrm{~h}$. These were then treated with 0.0625 and $0.125 \%$ CHD-FA for $24 \mathrm{~h}$. Negative controls were included. Metabolic activity of treated cells was then quantified using the XTT assay. Four isolates were used for each assay, and this was performed on two independent occasions in quadruplicate. ${ }^{*} p<0.05,{ }^{*} p<0.01,{ }^{* *} p<0.001$.
Initial studies aimed to evaluate the activity of CHD-FA and the three major classes of antifungal agent, namely azoles, polyenes, and echinocandins, using a panel of clinical C. albicans strains. This was primarily to assess the anti-biofilm activity of each drug compared to planktonic cells rather than direct antifungal drug comparison. CHD-FA was shown to kill rapidly and 
A

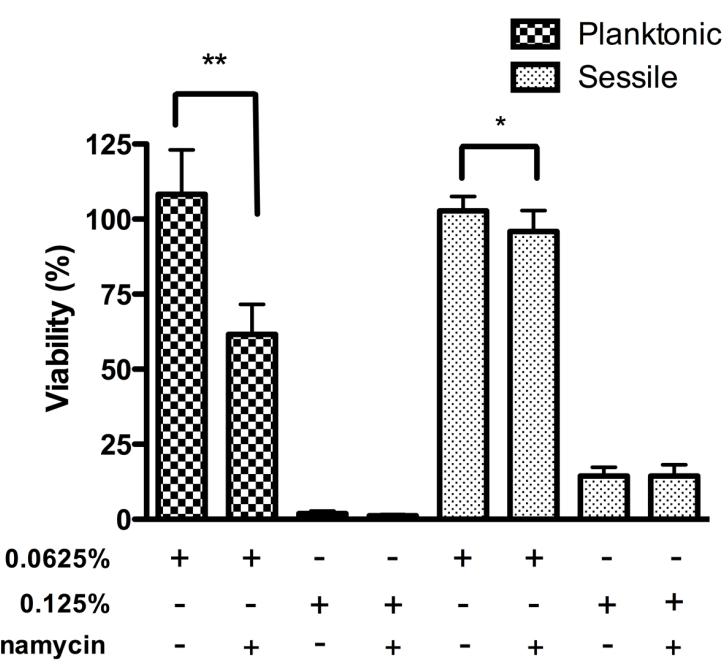

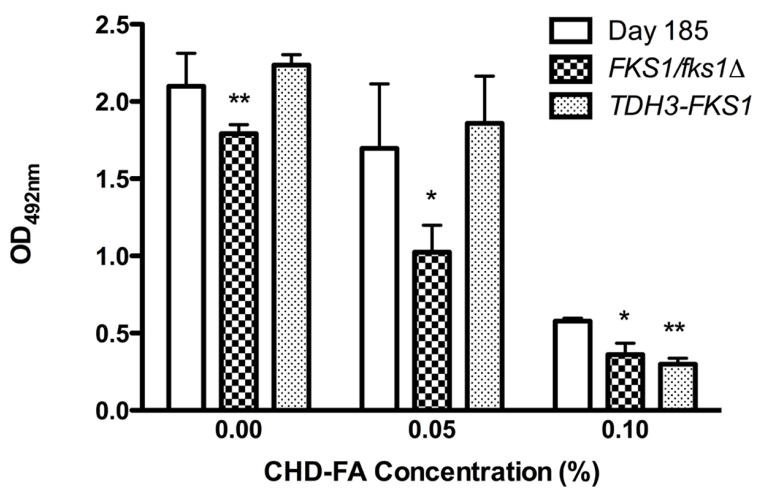

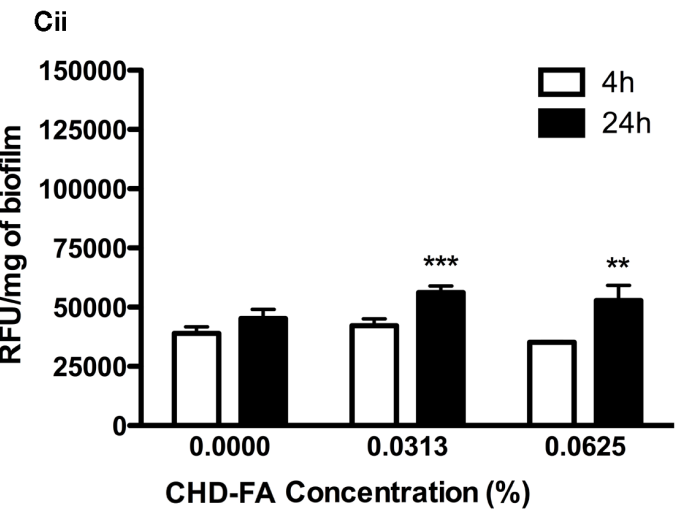

CHD-FA Concentration (\%)
$\mathrm{Ci}$

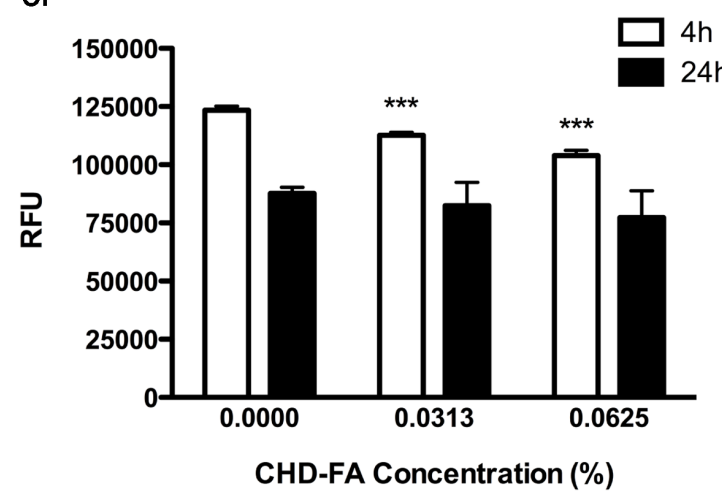

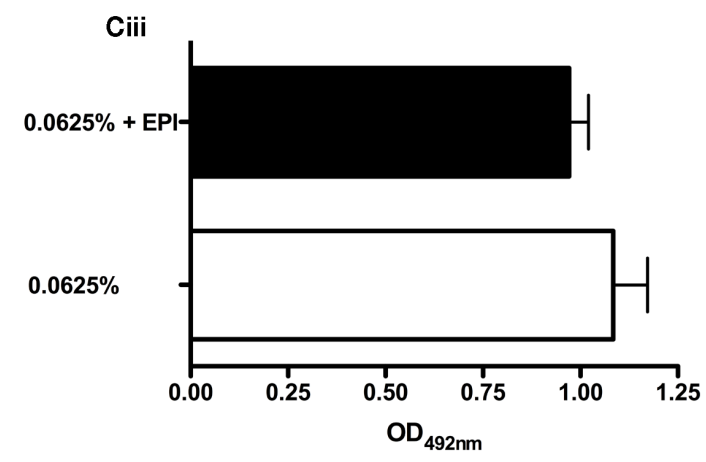

FIGURE 3 | Candida albicans biofilm resistance mechanisms play a limited role in CHD-FA sensitivity. (A) C. albicans was grown as planktonic and biofilm cells \pm geldanamycin $(12.5 \mu \mathrm{g} / \mathrm{ml})$ for $24 \mathrm{~h}$. These were then treated with 0.0625 and $0.125 \%$ CHD-FA for $24 \mathrm{~h}$. Negative controls were included. Metabolic activity of treated cells was then quantified using the XTT assay. Four isolates were used for each assay, and this was performed on two independent occasions in quadruplicate. (B) C. albicans DAY185, FKS1/fks1 $\triangle$ and TDH3-FKS1 were grown as biofilms for $8 \mathrm{~h}$ and treated with 0.05 and $0.1 \%$ CHD-FA for $24 \mathrm{~h}$. Negative controls were included. Metabolic activity of treated cells was then quantified using the XTT assay. This was performed on two independent occasions in triplicate. Statistical significance represents the comparison of FKS1/fks1 $1 \Delta$ to the reference strain DAY185. (C) C. albicans SC5314 was grown as (i) planktonic and (ii) biofilm cells and treated with 0.0313 and $0.0625 \%$ CHD-FA (sub-MIC levels) for 4 and $24 \mathrm{~h}$. Cells were washed, resuspended in assay buffer and $100 \mu \mathrm{g} / \mathrm{ml}$ of Ala-Nap added. These were then transferred to a black 96 -well plate for quantification at $30 \mathrm{~s}$ intervals over $60 \mathrm{~min}$ in a fluorescent plate reader $\left(\mathrm{Ex}_{355} / \mathrm{Em}_{460}\right)$. For biofilms the relative fluorescence is presented, which is normalized to dry weight of biofilms (RFU/mg). High fluorescence values indicate low efflux activity and vice versa. C. albicans $24 \mathrm{~h}$ biofilms were treated with $0.0625 \%$ $\mathrm{CHD}-\mathrm{FA} \pm \mathrm{EPI}$ for $24 \mathrm{~h}$ (iii). Biofilms were washed and metabolic activity measured using the XTT metabolic assay with absorbance read at $492 \mathrm{~nm}$. ${ }^{*} p<0.05,{ }^{* *} p<0.01,{ }^{* *} p<0.001$. be similarly effective against both planktonic and sessile cells for all strains, whereas the existing antifungal agents exhibited increased resistance in the biofilm phenotype. This is in agreement with earlier studies by our group and others who reported 
high level azole resistance and decreasing sensitivity of polyenes and echinocandins (Kuhn et al., 2002).

Disruption of the biofilm was also assessed, but despite CHDFA being highly fungicidal no significant reduction of the biofilm biomass was observed. Nevertheless, given the effectiveness of this compound then it would be possible to combine CHD-FA with a known C. albicans biofilm disrupting compound, such as lauroyl glucose (Dusane et al., 2008). Lauroyl glucose is a sugar ester that exhibits antimicrobial and emulsification properties (Ferrer et al., 2005), which contribute to the disruption of preformed C. albicans biofilms by up to $45 \%$ (Dusane et al., 2008). Therefore, these and other novel biofilm disruption agents could possibly be used to augment the activity of CHD-FA.

The use of SEM demonstrated the appearance of crenated cells, both planktonically and in biofilms. Visually, these appear similar to polyene treated cells, which integrates into and actively disrupts cell membranes, lysing their cytoplasmic contents (Gale, 1963). We therefore hypothesized that CHD-FA activity was potentially based on interaction with the cell membrane; an idea supported from the activity of CHD-FA against bacteria (van Rensburg et al., 2000). Membrane permeabilization assays were therefore used to confirm our hypotheses.

The measurement of intracellular ATP release has been previously described as an appropriate method to measure membrane permeability, as ATP leakage is not observed in viable cells (Smelt et al., 1994). We showed ATP release occurred in a steady manner with maximum release being observed after $40 \mathrm{~min}$ CHD-FA exposure, with a slight reduction observed thereafter; a similar phenomenon to a previous report (Smelt et al., 1994). PI uptake was in agreement with ATP release, again showing a time dependent disruption of the cell membrane. We demonstrated that $C$. albicans membrane integrity is compromised rapidly after exposure to CHD-FA, data supported visually from our fluorescent images showing PI uptake in as little as $1 \mathrm{~min}$ post-exposure (Ahmad et al., 2011).

To further test our cell membrane hypothesis we weakened the cell wall by inhibiting chitin biosynthesis using the chitin synthase inhibitor NKZ, which has been shown to inhibit all three Chs isozymes in C. albicans, and in turn results in a lack of chitin in fungal cells (Kim et al., 2002). We reasoned that if the membrane were the active target then the weakened cell would become hyper-susceptible to CHD-FA. We demonstrated that NKZ in combination with CHD-FA made both planktonic and sessile cells more susceptible to CHD-FA, which is similar to previous work showing that NKZ in combination with azoles increased $C$. albicans biofilm susceptibility (Kaneko et al., 2010).

Our group, amongst others, have shown the importance of biofilms in antimicrobial resistance, a multifactorial process (Ramage et al., 2009). We therefore aimed to evaluate the potential effects of key biofilm resistance mechanisms on CHD-FA. The major regulator of stress in C. albicans is Hsp90, which has been shown to potentiate the emergence and maintenance of resistance to azoles and echinocandins in C. albicans biofilms, at least in part via calcineurin (Robbins et al., 2011). Our study has also shown that Hsp90 plays a role in stress induced resistance, as planktonic and sessile C. albicans cells were more susceptible to CHD-FA at a sub-MIC level when geldanamycin, the Hsp90 inhibitor, is used in combination. Therefore, stress induced resistance mechanisms appear to play a role in C. albicans response to CHD-FA, however, this is more apparent in planktonic cells.

We next investigated the role of biofilm ECM, a key component and defining characteristic of fungal biofilms (Baillie and Douglas, 2000). Recent work by the Andes group has shown that ECM $\beta-1,3$ glucan, synthesized from Fks1p, is responsible for sequestering antifungal drugs and acting as a "drug sponge" (Nett et al., 2010a,b), therefore conferring resistance on C. albicans biofilms. Therefore, we aimed to determine whether CHD-FA was sequestered by ECM $\beta-1,3$ glucan in the same way as other classes of antifungal agents. For this we utilized strains used in the previous investigations (Nett et al., 2010b), where it was shown that $F K S 1 / f k s 1 \Delta$ biofilm sensitivity to fluconazole was exquisite in comparison to the biofilm resistant parental strain and the ECM $\beta-1,3$ glucan over-expressing strain TDH3-FKS1, which were highly resistant to fluconazole. In our investigations we have shown the FKS1/fks1 $\triangle$, TDH3-FKS1 and the reference strain, DAY185, were equally sensitive at $1 \times \mathrm{MIC}_{50}$ to CHD-FA. This is surprising given that Nett et al. (2010a) have shown that ECM impacts other antifungal classes. This suggests in our study that ECM $\beta-1,3$ glucan does not sequester CHD-FA, unlike azoles, polyenes, and echinocandins (Nett et al., 2010a).

Finally, we investigated whether efflux pumps are affected by CHD-FA, as these have previously been shown to be expressed in C. albicans biofilms (Ramage et al., 2002). In this study we showed efflux pump activity in both planktonic and sessile C. albicans cells, but only significant up-regulation after $4 \mathrm{~h}$ treatment in planktonic cells at sub-MIC levels of CHD-FA. However, overall efflux pump activity in C. albicans biofilms was found to be higher when compared to planktonic cells, which was predicted based on previous literature (Ramage et al., 2002). In order to determine their contribution to potential resistance to CHD-FA we inhibited efflux pump activity using a competitive substrate. Efflux pump inhibitor studies have previously shown, with the use of mutants with single and double deletion mutations, that $C$. albicans have reduced mRNA expression for various multidrug resistant efflux pumps (Ramage et al., 2002). Here we showed that this compound did not alter the MIC. These data indicates that CHD-FA is not impacted by efflux pump activity, despite the fact that these pumps were induced through treatment. This is unsurprising given that CHD-FA acts on the cell membrane and not through an internal cellular mechanism.

CHD-FA is a microbicidal compound that acts non-specifically on the cell membrane. Therefore, it may serve as a potential novel antiseptic agent for the treatment of oral candidosis and other candidal biofilm infections. Increasingly Candida biofilms are becoming clinically important (Tumbarello et al., 2007; Ramage et al., 2009), particularly because of inappropriate or misuse of broad spectrum antimicrobials that are ineffective against recalcitrant biofilms (Niimi et al., 2010). In the context of oral health, individuals suffer from a range of oral candidal diseases that cause high levels of morbidity. Moreover, there are many other oral diseases of microbial biofilm origin, such as caries and periodontitis, which would benefit from novel antiseptic molecules, assuming these meet appropriate safety standards. CHD-FA has already shown to have no sign of toxicity in rats and humans, and in 
fact was reported to have anti-inflammatory and wound healing properties, possibly through a free radical scavenging mechanism (Gandy et al., 2011; Sabi et al., 2011). Whilst CHD-FA appears to have appropriate biological properties of an antiseptic, further cellular and in vivo studies are required.

\section{REFERENCES}

Ahmad, A., Khan, A., Kumar, P., Bhatt, R. P., and Manzoor, N. (2011). Antifungal activity of Coriaria nepalensis essential oil by disrupting ergosterol biosynthesis and membrane integrity against Candida. Yeast 28, 611-617.

Baillie, G. S., and Douglas, L. J. (2000). Matrix polymers of Candida biofilms and their possible role in biofilm resistance to antifungal agents. J. Antimicrob. Chemother. 46, 397-403.

Clinical Laboratory Standards Institute (CLSI). (2008). Reference Method for Broth Dilution Antifungal Susceptibility Testing of Yeast, Approved Standard - 3rd Edn, CLSI document M27-A3. Wayne, PA: CLSI.

Coco, B. J., Bagg, J., Cross, L. J., Jose, A., Cross, J. G., and Ramage, G. (2008). Mixed Candida albicans and Candida glabrata populations associated with the pathogenesis of denture stomatitis. Oral Microbiol. Immunol. 23, 377-383.

Dusane, D. H., Rajput, J. K., Kumar, A. R., Nancharaiah, Y. V., Venugopalan, V. P., and Zinjarde, S. S. (2008). Disruption of fungal and bacterial biofilms by lauroyl glucose. Lett. Appl. Microbiol. 47, 374-379.

Erlandsen, S. L., Kristich, C. J., Dunny, G. M., and Wells, C. L. (2004). High-resolution visualization of the microbial glycocalyx with low-voltage scanning electron microscopy: dependence on cationic dyes. J. Histochem. Cytochem. 52, 1427-1435.

Ferrer, M., Soliverib, J., Ploua, F. J., López-Cortésa, N., Reyes-Duartea, D., Christensenc, M., Copa-Patiñob, J. L., and Ballesterosa, A. (2005). Synthesis of sugar esters in solvent mixtures by lipases from Thermomyces lanuginosus and Candida antarctica $\mathrm{B}$, and their anti-microbial properties. Enzyme Microb. Technol. 36, 391-398.

Gale, G. R. (1963). Cytology of Candida albicans as influenced by drugs acting on the cytoplasmic membrane. J. Bacteriol. 86, 151-157.

Gandy, J. J., Snyman, J. R., and Van Rensburg, C. E. (2011). Randomized, trolled study to evaluate the efficacy and safety of carbohydrate-derived fulvic acid in topical treatment of eczema. Clin. Cosmet. Investig. Dermatol. 4, 145-148.

Jose, A., Coco, B. J., Milligan, S., Young, B., Lappin, D. F., Bagg, J., Murray, C., and Ramage, G. (2010). Reducing the incidence of denture stomatitis: are denture cleansers sufficient? J. Prosthodont. 19, 252-257.

Kaneko, Y., Ohno, H., Fukazawa, H., Murakami, Y., Imamura, Y., Kohno, S., and Miyazaki, Y. (2010). AntiCandida-biofilm activity of micafungin is attenuated by voriconazole but restored by pharmacological inhibition of Hsp90-related stress responses. Med. Mycol. 48, 606-612.

Kim, M. K., Park, H. S., Kim, C. H., Park, H. M., and Choi, W. (2002). Inhibitory effect of nikkomycin $\mathrm{Z}$ on chitin synthases in Candida albicans.

Kuhn, D. M., George, T., Chandra, J., Mukherjee, P. K., and Ghannoum, M. A. (2002). Antifungal susceptibility of Candida biofilms: unique efficacy of amphotericin B lipid formulations and echinocandins. Antimicrob. Agents Chemother. 46, 1773-1780.

Nett, J. E., Crawford, K., Marchillo, K., and Andes, D. R. (2010a). Role of Fkslp and matrix glucan in Candida albicans biofilm resistance to an echinocandin, pyrimidine, and polyene. Antimicrob. Agents Chemother. 54, 3505-3508.

Nett, J. E., Sanchez, H., Cain, M. T., and Andes, D. R. (2010b). Genetic basis of Candida biofilm resistance due to drug-sequestering matrix glucan. J. Infect. Dis. 202, 171-175.

Niimi, M., Firth, N. A., and Cannon, R. D. (2010). Antifungal drug resistance of oral fungi. Odontology 98, 15-25.

Pierce, C. G., Uppuluri, P., Tristan, A. R., Wormley, F. L. Jr., Mowat, E., Ramage, G., and Lopez-Ribot, J. L. (2008). A simple and reproducible 96-well plate-based method for the formation of fungal biofilms and parallel-group, double-blind, conYeast 19, 341-349.

\section{ACKNOWLEDGMENTS}

We are grateful to Fulhold for providing CHD-FA and supporting the studentship of Leighann Sherry. We would like to acknowledge David Andes and his group (University of Wisconsin) for providing the FKS1 strains.

its application to antifungal susceptibility testing. Nat. Protoc. 3, 1494-1500.

Ramage, G., Bachmann, S., Patterson, T. F., Wickes, B. L., and Lopez-Ribot, J. L. (2002). Investigation of multidrug efflux pumps in relation to fluconazole resistance in Candida albicans biofilms. J. Antimicrob. Chemother. 49, 973-980.

Ramage, G., Culshaw, S., Jones, B., and Williams, C. (2010). Are we any closer to beating the biofilm: novel methods of biofilm control. Curr. Opin. Infect. Dis. 23, 560-566.

Ramage, G., Jose, A., Coco, B., Rajendran, R., Rautemaa, R., Murray, C., Lappin, D. F., and Bagg, J. (2011a). Commercial mouthwashes are more effective than azole antifungals against Candida albicans biofilms in vitro. Oral Surg. Ora Med. Oral Pathol. Oral Radiol. Endod. 111, 456-460.

Ramage, G., Rajendran, R., GutierrezCorrea, M., Jones, B., and Williams, C. (2011b). Aspergillus biofilms: clinical and industrial significance. FEMS Microbiol. Lett. 324, 89-97.

Ramage, G., Mowat, E., Jones, B. Williams, C., and Lopez-Ribot, J. (2009). Our current understanding of fungal biofilms. Crit. Rev. Microbiol. 35, 340-355.

Ramage, G., Vande Walle, K., Wickes, B. L., and Lopez-Ribot, J. L. (2001). Standardized method for in vitro antifungal susceptibility testing of Candida albicans biofilms. Antimicrob. Agents Chemother. 45, 2475-2479.

Robbins, N., Uppuluri, P., Nett, J., Rajendran, R., Ramage, G., Lopez-Ribot, J. L., Andes, D., and Cowen, L. E. (2011). Hsp90 governs dispersion and drug resistance of fungal biofilms. PLoS Pathog. 7, e1002257. doi:10.1371/journal.ppat.1002257

Sabi, R., Vrey, P., and Van Rensburg, C. E. (2011). Carbohydrate-derived fulvic acid (CHD-FA) inhibits carrageenan-induced inflammation and enhances wound healing: efficacy and toxicity study in rats. Drug Dev. Res. 73, 18-23.

Samaranayake, L. P., Keung Leung, W. and Jin, L. (2009). Oral mucosal fungal infections. Periodontol. 2000 , 49, 39-59.

Smelt, J. P. P. M., Rijke, A. G. F., and Hayhurst, A. (1994). Possible mechanism of high pressure inactivation of microorganisms. High Press. Res. 12, 199-203.

Tumbarello, M., Posteraro, B., Trecarichi, E. M., Fiori, B., Rossi, M., Porta, R., De Gaetano Donati, K., La Sorda, M., Spanu, T., Fadda, G., Cauda, R., and Sanguinetti, M. (2007). Biofilm production by Candida species and inadequate antifungal therapy as predictors of mortality for patients with candidemia. J. Clin. Microbiol. 45, 1843-1850.

van Rensburg, C. E., Van Straten, A., and Dekker, J. (2000). An in vitro investigation of the antimicrobial activity of oxifulvic acid. J. Antimicrob. Chemother. 46, 853.

Conflict of Interest Statement: The authors declare that the research was conducted in the absence of any commercial or financial relationships that could be construed as a potential conflict of interest.

Received: 17 February 2012; paper pending published: 27 February 2012; accepted: 12 March 2012; published online: 29 March 2012.

Citation: Sherry L, Jose A, Murray C, Williams $C$, Jones B, Millington $O$, Bagg $J$ and Ramage $G$ (2012) Carbohydrate derived fulvic acid: an in vitro investigation of a novel membrane active antiseptic agent against Candida albicans biofilms. Front. Microbio. 3:116. doi: 10.3389/fmicb.2012.00116

This article was submitted to Frontiers in Antimicrobials, Resistance and Chemotherapy, a specialty of Frontiers in Microbiology.

Copyright (C) 2012 Sherry, Jose, Murray, Williams, Jones, Millington, Bagg and Ramage. This is an open-access article distributed under the terms of the Creative Commons Attribution Non Commercial License, which permits noncommercial use, distribution, and reproduction in other forums, provided the original authors and source are credited. 